\section{Dendritic cell dysfunction and implications for dendritic cell-based therapy in colorectal cancer}

\author{
Michael Vallop Josiassen, ${ }^{1}$ \\ Kajsa Paulsson ${ }^{2}$ \\ ${ }^{1}$ Laboratory of Experimental \\ Immunology, Institute of International \\ Health, Immunology and Microbiology, \\ University of Copenhagen, Copenhagen, \\ Denmark; ${ }^{2}$ Department of Experimental \\ Medical Sciences, Immunology Section, \\ Lund University, Lund, Sweden
}

\begin{abstract}
Clinical trials and experimental models indicate that dendritic-cell-based immunotherapy is promising for treatment of different types of cancer. However, dendritic cells (DCs) have strong immune-regulatory capacities and can not only stimulate but also dampen immune responses. It is also well known that the different DC subsets strongly influence the magnitude and quality of adaptive immune responses. In this review, to improve understanding of the DC-based immunotherapy approach, we briefly describe different DC subsets and the differentiation, maturation, and activation of these cells. One form of cancer for which there is a strong need to find, establish, and standardize new, alternative therapies is colorectal cancer. This review discusses some of the factors, including those involved in DC dysfunction, that we believe to be of major influence in DC therapy in colorectal cancer.
\end{abstract}

\section{Introduction}

For a tumor to progress and become fatal it must evade the immunosurveillance of the host and the mechanisms by which malignant cells are destroyed. Activation of the body's own immune defense to destroy tumor cells has been a goal for many years. Promising results have been achieved in clinical trials, e.g. by blocking the T cell-expressed negative immune modulator CTLA-4. ${ }^{1-3}$ A challenge in anti-CTLA-4 therapy is to avoid the potential risk of inducing autoimmune effects. Another focus for potential immunotherapy has been and is the dendritic cell (DC). The DC is of crucial importance in recognizing and presenting pathogens, and is often referred to as nature's adjuvant due to its unique ability to capture, process, and present antigens which in turn stimulate a specific immune response. ${ }^{4}$ The importance of DCs in cancer has been known for more than 20 years, and it has been shown that many patients with tumors invaded by DCs have a far better prognosis. ${ }^{5}$ Other studies have shown that DCs isolated from cancer patients have a significantly lower effect in stimulating an immune response than do DCs isolated from healthy patients, and even T cells from healthy patients seem to perform worse in a tumor-induced environment.6,7 This implies that the tumor environment limits the effect of DCs; correcting this might first of all give us an important insight in how tumors escape immune-surveillance, but it might also help to improve treatment against cancer.

Under normal circumstances DCs are only found in small amounts in the human organism, and this has somewhat limited the evolution of DC vaccines. However, progress in the in vitro production of DCs has now made it easier and less costly to produce amounts that allow their use in therapeutic settings. ${ }^{8}$ This development, in combination with the continuing discovery of different tumor antigens and knowledge of how to modify these antigens beneficially in vaccines, has evoked increased interest in DC-based therapy. A valid reason to use this kind of immunologic approach is that in a number of cancers the normal antigen presentation and maturation of DCs are frequently defective but can be to some extent bypassed in DC-based therapy: by targeting tumor antigens to DCs in vitro or in vivo, the process of antigen presentation and maturation can be regulated. However, a major challenge for successful DC-based cancer therapy is overcoming the tumor-microenvironmentinduced immune suppression.

Colorectal cancer (CRC) is a type of cancer for which there is a dire need of alternative treatments besides surgery, radiotherapy, and chemotherapy. Radio- and chemotherapy have been strongly associated with both a poor quality of life for the patient and adverse side effects. Systemic toxicity is not the only problem; there is also the question of the development of tolerance seen with chemotherapy. Last but not least, there is the fact that $30 \%$ of newly diagnosed CRC patients have unresectable tumors, and $19 \%$ of patients have developed metastases at the time of initial diagnosis. ${ }^{9-11}$

In general, CRC can be divided into three groups according to hereditary predisposition and cancer risk: i): sporadic CRC with no inherited predisposition (60\%); ii) familial CRC with at least one affected blood relative but no inherited genetic mutation (30\%); and, finally, iii) hereditary CRC syndromes (10\%). ${ }^{12}$ In CRC the tumor is often genetically unstable: alterations of the genes involved in antigen presentation and the HLA class I expression often occur, ${ }^{13-15}$ and CRC can be further subdivided on the basis of whether the tumor con-
Correspondence: Kajsa Paulsson, Department of Experimental Medical Sciences, Immunology Section, BMC-D14, Lund University, SE-221 84 Lund, Sweden.

Tel: +46.46.222.4167.

E-mail: kajsa_m.paulsson@med.lu.se

Key words: dendritic cell dysfunction, colorectal cancer.

Acknowledgments; we thank Camilla Thuring and Hans-Olov Sjögren for critical reading of the manuscript, and help with the illustrations. We thank for financial support: Novo Nordisk Foundation, Lundbeck Foundation, WedellWedellsborgs Foundation, Benzon Foundation, Copenhagen Cluster of Immunology, Swedish Medical Research Council, Crafoord Foundation, Royal Physiographic Society in Lund, Thelma Zoegas, Magnus Bergvalls, Groshinskys, Greta and Johan Kocks Foundations.

Received for publication: 27 June 2011. Accepted for publication: 13 September 2011.

This work is licensed under a Creative Commons Attribution NonCommercial 3.0 License (CC BYNC 3.0).

(C) Copyright M. Vallop Josiassen and K. Paulsson, 2011 Licensee PAGEPress, Italy

Drugs and Therapy Studies 2011; 1:e14

doi:10.4081/dts.2011.e14

tains a mutation resulting in microsatellite instability (MSI). MSI occurs because the mutation leads to a defect in the DNA mismatch repair system, with subsequent accumulation of deletions and insertions of single nucleotides in repetitive DNA sequences. ${ }^{15}$ The MSI phenotype is characteristic in most hereditary non-polyposis colorectal cancers (HNPCC) but is also seen in $10-30 \%$ of sporadic CRCs. ${ }^{12,16}$ For a functional $\mathrm{CD} 8+\mathrm{T}$ cell immune response to take place against a tumor cell, antigens should be presented by HLA class I molecules on the surface of the cell. In 1998 Cabrera et al. showed that $73 \%$ of the cases in a study of 78 CRC patients had detectable HLA class I deficiency. ${ }^{14}$ Other studies have proposed that the different types of CRC might follow individual routes in loss of HLA class I complexes as a result of whether MSI is present or not. Defective HLA class I expression in MSI-positive tumors may be due to alteration of the $\beta 2$-microglobulin molecule, but loss of HLA class I in MSI-negative tumors might be an effect of modulation of antigenprocessing machinery (APM) components such as TAP and/or tapasin. ${ }^{16,17}$ Recently a distinction between the loss of HLA class I in sporadic MSI-positive and HNPCC MSI-positive tumors was discovered by Dierssen et al. ${ }^{18}$ Sporadic MSI-positive tumors were associated with defects in the APM, whereas the loss of 
HLA class I molecules in the HNPCC MSI-positive tumors were correlated with defects in the $\beta 2$-microglobulin expression. In addition to antigen presentation on tumor cells per se, efficient professional antigen presentation (i.e., priming of naïve $\mathrm{T}$ cells by $\mathrm{DCs}$ ) must take place.

The purpose of this paper is to critically review current knowledge about how DC function is inhibited in CRC, with a special focus on how knowledge about tumor evasion might help to improve the generation in the future of the different types of recently developed DCbased therapies.

\section{DC differentiation, maturation and activation}

To fully comprehend the strategies of DCbased therapy and how colorectal tumors might evade it, a basic understanding of the DCs is necessary. DCs are professional antigen-capturing and presenting cells that efficiently process proteins and present peptides fragments on both MHC class I and II molecules along with appropriate co-stimulatory molecules. The progenitor DCs are distributed through the blood circulation to peripheral tissues and are found under most surface epithelia and in solid organs. In this state they are not yet able to mobilize or elicit immune responses, which is why they are called immature DCs. The primary function of these cells is to scan peripheral tissues and lymphoid organs for any antigens, which they engulf through pinocytosis, phagocytosis, or receptor-mediated mechanisms. The types of antigens that can be captured may be products or debris made by viruses, bacteria, or even tumor material.

A process called cross-presentation allows DCs to take up an extracellular antigen and present part of it on MHC class I molecules, which in other cells are usually restricted to presenting intracellular antigens. ${ }^{19}$ After antigen capture and processing, the DC matures and migrates to a draining lymph node, where it eventually interacts with naïve $\mathrm{T}$ cells. The DC enters a draining lymph node from the afferent lymphatics. CCR7 is considered a gatekeeper chemokine receptor that is induced during DC maturation and is required for the migration of DCs from the periphery to the lymph nodes. ${ }^{20,21}$ This receptor is specifically sensitive to the CCL19 and/or CCL21 chemokines produced by lymph node tissue and not only initiates migration but is also thought to contribute to the further maturational stages. ${ }^{22-24}$ When entering the lymph node the DC has already made the transition to a mature non-phagocytosing $\mathrm{DC}$ and expresses vast amounts of long-lived HLA molecules and diverse adhesion and co-stimulatory molecules, making the DC a very potent antigenpresenting cell. The mature DC is less efficient at capturing and processing further antigens, but on the other hand it is highly efficient at $\mathrm{T}$ cell activation and co-stimulation. By presenting tumor-derived antigens to naïve T cells the DC activates and stimulates clonal expansion, allowing a specific immune response against the tumor. Nevertheless, several tumor-derived products in CRC, together with downregulation of HLA molecules, create an unique tumor microenvironment that prohibits DCs from presenting antigens in a way that elicits antitumor responses.

The development and regulation of human DCs is only marginally understood. ${ }^{25}$ Translation to human from mouse models has been difficult because the relationship between mouse and human DC subsets remains in part elusive. CD $34^{+}$progenitor cells can differentiate into distinct dendritic precursor cells and form two major types of DC lineages: conventional DCs (cDCs), also known as myeloid DCs (mDCs), and plasmacytoid DCs (pDCs), sometimes called lymphoid DCs (IDCs).$^{26} \mathrm{~A}$ common conception has been that cDCs originate from a myeloid progenitor and pDCs from a lymphoid progenitor. However, evidence has been presented in mice for generation of both $\mathrm{cDCs}$ and $\mathrm{pDCs}$ from both common lymphoid and myeloid progenitors. ${ }^{27}$ In addition, it has been shown that a common

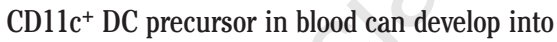
both pDCs and cDCs. ${ }^{28} \mathrm{cDCs}$ further differentiate into interstitial DCs, dermal DCs, and Langerhans DCs. pDCs look similar to plasma cells, but share particular features with cDCs. ${ }^{29}$ Other characteristics are not shared with either plasma cells or cDCs, i.e., they can produce large amounts of type I interferon $(\alpha, \beta$, $\omega)$ in response to viral and bacterial infections. ${ }^{1,30}$ After activation, pDCs differentiate into mature DCs that exhibit dendritic form and antigen-presenting functions, hence regulating the functions of $\mathrm{T}$ cells and forming a functional link between innate and adaptive immune responses. ${ }^{31}$ In contrast to the DCs, which originate from hematopoietic progenitors, follicular DCs (FDC) ${ }^{32}$ are probably of mesenchymal origin and will not be further discussed here.

The DCs can be impaired through specific mechanisms such as decreased generation, function inhibition, polarization of the DC subsets into inactive cells, or prevention of the DCs from coming into contact with naïve $T$ cells. All of these mechanisms result in a lack of $\mathrm{T}$ cell stimulation and hence no specific immune response against the tumor cells.

Knowledge obtained from tumor evasion mechanisms in CRC now provides the opportunity to specifically design improved $\mathrm{DC}$ vaccines, trying to make them as unaffected as possible by immunosuppressing tumor-derived products while still inducing a potent immune response. For this to succeed, several parameters, both in the vaccine production and in the tumor environment, need to be investigated and characterized before they can be optimized and controlled.

\section{Dendritic cell dysfunction in CRC}

Numerous studies have shown that tumor development is strongly influenced by its microenvironment. ${ }^{33} \mathrm{~A}$ tumor-supportive environment is created by several different cells including both stromal and non-malignant cells. In many tumor microenvironments macrophages are the most frequent immune cell, and macrophage blood precursors have been suggested to be recruited by the tumorcell-produced CCL2 chemokine, VEGF, PDGF, TGF $\beta$ and M-CSF. ${ }^{34,35}$ The tumor-associated macrophages play an important role in tumorigenesis and have in some models been shown to be necessary for the formation of metastases. ${ }^{36}$ Other immune cells in the tumor microenvironment include T cells, NK cells, granulocytes, monocytes, and DCs. ${ }^{37}$ A group of myeloid cells that in mice can be distinguished at an early stage of differentiation because of their CD11b and Grl expression are called myeloid-derived suppressor cells (MDSCs) ${ }^{38}$

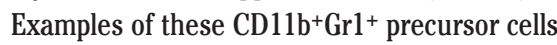
are immature DCs, immature macrophages, and immature granulocytes. Under normal circumstances the $\mathrm{CD} 1 \mathrm{~b}^{+} \mathrm{Grl}^{+}$precursor cells can differentiate into healthy cells such as mature DCs, but an alteration in the cytokine and chemokine balance due to tumor growth, infection, or immune stress may lead to their suppressive phenotype and the induction of tolerance in peripheral organs. Some of the tumor-derived products involved in the regulation of MDSC generation, recruitment, and activation are CSF-1, IL-6, VEGF, IL-10, GMCSF, IL-13, IFN- $\gamma$, and PGE. They primarily trigger signaling pathways on MDSCs that involve the Janus kinase (JAK) protein family that activates the main transcription factor STAT3, which in turn regulates MDSC expansion. Some of the tumor-derived products implicated in MDSC immune suppression will be described below in more detail, but several others exist that are not included in the scope of this paper (e.g., TGF- $\beta$, arginase 1 , iNOS, ROS), and in CRC a crucial link has been suggested between the immunosuppressive tumor environment and MDSCs. ${ }^{39}$

DCs are modified by the tumor microenvironment through several mechanisms that protect the tumor from recognition by $\mathrm{T}$ cells. These can in general be divided into four categories: i) elimination of DC function by impairment of antigen capture, processing, 
and presentation or lack of production/differentiation/maturation; ii) induction of apoptosis in DCs; iii) polarization of DC subpopulations into immunosuppressive or tolerogenic cells; iv) avoiding attraction of DCs to the tumor site by downregulation of DC-attracting chemokines. Multiple stroma- and tumorderived factors have been recognized to have the potential to alter the DCs at tumor sites or systemically. We describe here a subset of factors with DC-altering mechanisms representing what we think is, from a DC point of view, a key set of stroma- and tumor-derived factors in CRC.

\section{Vascular endothelial growth factor}

Vascular endothelial growth factor (VEGF) is a homodimeric glycoprotein produced in normal cells especially during embryogenesis and neovascularization, but it is also a well-known tumor-derived product. ${ }^{40}$ It possesses the ability to develop new blood vessels which are necessary for a tumor growth of more than $2 \mathrm{~mm}$, but it can also inhibit DC maturation by acting on $\mathrm{CD}^{+} 4^{+}$hematopoietic progenitor cells. VEGF has been shown to block generation of DCs by binding to the Flt-1 receptor and thus stop further activation of the transcription factor NF-кB. This was discovered from in vivo murine studies in which administration of recombinant VEGF showed significant reduction of DC generation. ${ }^{41}$ Another study showed that VEGF leaves the $\mathrm{CD} 4^{+}$hematopoietic progenitor cell unable to differentiate further and the DC production stops. ${ }^{42}$ The effect of VEGF on DCs was fully verified in vitro when anti-VEGF antibody applied in a tumor-cellconditioned medium with CD $34^{+}$hematopoietic progenitor cells led to full generation of DCs. ${ }^{43}$

Gabrilovich et al. suggested that the H1 histone gene was implicated in the malfunction of DC production and was inhibited by several tumor-derived products including VEGF. Mouse specimens lacking the $\mathrm{H} 1$ histone gene were found to have a significantly lower production of DCs but a normal amount of serum macrophages, granulocytes, and lymphocytes. They further demonstrated that $\mathrm{H} 1$ histone expression was regulated through the transcription factor NF- $\kappa \mathrm{B}$, a transcription factor blocked by VEGF. This fact led to the hypothesis that the $\mathrm{H1}$ histone might play a pivotal role in the regulation of $\mathrm{DC}$ generation further down the NF-кB pathway. ${ }^{4}$

\section{Interleukin-10}

Interleukin-10 (IL-10) is another cytokine important in CRC and is well known to be an immunosuppressant resulting in insufficiencies in antigen presentation, $\mathrm{T}$ cell proliferation, and Th1 cytokine secretion. Several different models have been used to analyze the role of IL-10 in different cancers, and depending on the model used either a tumor-promoting or a tumor-inhibiting effect has been seen. ${ }^{45}$ IL-10 directly inhibits both the generation and differentiation of DCs. ${ }^{46}$ DCs derived from transgenic mice with overexpression of IL-10 have a lower chance of stimulating allogeneic T cells, cytotoxic T lymphocyte (CTL) responses, and production of the protumorigenic cytokine IL-12. ${ }^{47}$ DC maturation and function depend on CD40/CD40 ligand interaction, and Shurin et al. showed that, in MC38 colon adenocarcinoma tumor models, DCs had a downregulation of the CD40 expression which was directly due to IL-10. The correlation was further supported by the use of antiIL-10 antibody, resulting in increased concentration of CD40-dependent IL-12.48 As mentioned above, the effect of IL-10 on tumor development varies, but several preclinical and clinical experiments have shown an antitumor effect and regression of tumors after IL-10 treatment. ${ }^{49,50}$

\section{Interleukin-8}

Interleukin-8 (IL-8) is a multifunctional member of the CXC chemokine superfamily. It is also a normal product of human colonic crypt cells and is thought to attract neutrophil granulocytes in inflammation and to promote angiogenesis to damaged vascular epithelial cells. ${ }^{51-53}$ Various neoplasms express IL-8 constitutively, and when colonic cells become malignant the IL- 8 production is much greater than in healthy tissue. In CRC IL-8 has been suggested to provide both autocrine growth and angiogenesis to malignant cells. An increasing body of evidence also indicates that in CRC IL-8 production is a hallmark of cancer progression and metastatic potential. ${ }^{54,55}$ AntiIL- 8 antibody can be used to inhibit cell division, implying that IL-8 is not the only stimulus in cell growth but certainly has an influence on proliferation..$^{51}$ A clinical trial suggested a link between IL- 8 and defective DCs. ${ }^{56}$ Patients suffering from cancer, including CRC, were given intratumoral injections of the antitumorigenic IL-12, and retention of DCs was found. Consequently these DCs were not able to migrate to lymph nodes and start an immune response. The common denominator of the cancer types was the elevated concentration of IL- 8 , and it was also found that the DCs expressed both the IL- 8 receptors CXCR1 and CXR2 on their cell surface. Neutralizing antibodies against IL-8 prevented infiltration and, most importantly, retention of DCs into the tumors, and hence the role of IL- 8 was suggested to be as a chemoattracting agent for DCs.

\section{Macrophage colony-stimulating factor and Interleukin-6}

Macrophage colony-stimulating factor (M-
CSF) or colony stimulating factor-1 (CSF-1) influences the survival, proliferation, and differentiation of hematopoietic stem cells (HSCs) into macrophages and DCs. ${ }^{57} \mathrm{M}-\mathrm{CSF}$ has been shown to be produced by colon carcinoma cells ${ }^{58}$ and IL-6, secreted by macrophages and T cells, has been shown to inhibit some of the later stages of CD34+ hematopoietic precursor cell differentiation. CD34+ hematopoietic precursor cells normally differentiate into various cells including DCs, but when M-CSF and IL-6 are produced in cancers they polarize the $\mathrm{CD} 34^{+}$hematopoietic precursor cells towards a non-DC-producing cell lineage ${ }^{59-61}$ A study of IL-6 knockout mice showed that these mice had increased amounts of mature DCs, suggesting that IL-6 might play a role in maintaining DCs at the immature stage. This study further showed that IL-6 activates the signal-transducing molecule STAT3, and that the IL-6 induced STAT3 pathway was indeed responsible for the inhibition of lipopolysaccharide-induced DC maturation. ${ }^{61}$

\section{Indoleamine 2,3-dioxygenase}

Indoleamine 2,3-dioxygenase (ID0) is an enzyme that in recent years has attracted much attention because it catalyzes the oxidative cleavage of the indole ring of several important regulatory molecules, including tryptophan..$^{62}$ Tryptophan is a key amino acid not only for pathogen proliferation but also for T-cell proliferation and differentiation. ${ }^{43}$ ID0 can be induced during an immune response in many cell types. ${ }^{63}$ In 2006 Brandacher et al. published results from an in vitro experiment in which different human CRC cell lines were tested for IDO expression. ${ }^{64}$ Without IFN- $\gamma$ none of the CRC lines expressed IDO, but when IFN- $\gamma$ was added they all expressed it. Subsequently the authors collected tumor tissue samples from 143 patients with primary CRC who were undergoing surgery. The results showed that in all 143 cases ID0 was expressed in tumor cells, and antigen-presenting cells such as macrophages and plasmacytoid DCs were also found to be ID0-positive. These authors further demonstrated an inverse correlation between tumor-infiltrating $\mathrm{T}$ cells and ID0 expression, and also showed a strong correlation between high ID0 expression and the presence of metastases.

IDO dysregulation has been shown in pDCs and was suggested to be a result of tumorderived stimulating factors. Regulatory T cells (T-regs) can upregulate IDO expression in antigen-presenting cells, including DCs, through binding to CTLA-4. ${ }^{65}$ Furthermore, $\mathrm{IDO}^{+} \mathrm{pDCs}$ in tumor-draining lymph nodes might recruit T-reg cells in a feed-forward loop whereby the T-reg cells suppress DC antigenpresenting function, impairing immunostimu- 
lation and resulting in immunosuppression of other T cells.

\section{Dendritic cell-based therapy}

\section{DC vaccine production}

DCs are present in relatively low amounts in the body and are not easy to isolate in amounts usable for immunotherapy. Instead, the three main sources of mDCs used in clinical trials are in vitro culture of peripheral blood $\mathrm{CD}^{+} 4^{+}$ monocytes, CD34+ hematopoietic progenitor cells, and circulating DC precursors, which, with the addition of certain key cytokines such as GM-CSF, TNF- $\alpha$, or IL-4, produce monocytederived DCs (Mo-DCs), CD34+-derived DCs, and peripheral-blood DCs (PBDCs), respective$\mathrm{ly}^{66-70}$ (Figure 1). This way of generating DCs also offers the distinct advantage of vaccine modification. Determining parameters such as DC subset, maturational status, antigen type, and cell surface activation molecules may lead to more specific antitumor therapy, and, most importantly, provides the possibility of bypassing the immunosuppressive factors caused by the tumor microenvironment. ${ }^{66}$ However, the large variability in vaccine protocols, relating to antigen type, dose, injection frequency, and route of administration, among others, has made it very complicated to compare and draw any firm conclusion from these studies. For this reason, the outcomes of $\mathrm{DC}$ vaccines in clinical trials will not be in focus in the following section, but rather various options in the fabrication of DC vaccines that may help avoid tumorinduced DC dysregulation.

\section{DC subset of choice}

Finding the most immunogenic and least tolerogenic DC subset has proven difficult, since most findings from murine studies are difficult to apply to the human setting. Comparative reports made have indicated that circulating myeloid DCs have the best possible migration to the lymph node but also have impaired secretion of immunostimulatory cytokines. CD34+-derived DCs are suggested to be more immunogenic and thus more capable of presenting the antigen of choice in a potential vaccine. However, since it has turned out that Mo-DCs are easier to manufacture they are the most commonly used subset in clinical trials. ${ }^{67}$

\section{Mature vs immature DCs}

Antigen presentation by DCs can lead to either tolerance or activation, and for this reason it is of great importance to understand this process in detail when an immune response with subsequent elimination of tumor cells is the goal. One factor of major impact is the maturational status of the DCs, since antigen presentation by immature DCs is poorly immunogenic and the antigen presentation by these cells to naïve T lymphocytes has in some cases indeed been shown to induce tolerance rather than activation ${ }^{68,69}$ (Figure 2). Maturation can easily be induced ex vivo by coculturing the DCs with a combination of inflammatory stimuli (TNF- $\alpha$, IL-6, IL-1 $\beta$, PGE2), and it is the mature DCs that are most often used in vaccine protocols.

\section{Type of antigen and loading technique}

Cultured DCs can be loaded with several different types of antigens in order to elicit a tumor-specific immune response, and the most common procedure has been loading HLA molecules with peptides from defined antigens. This presents several disadvantages, such as limited well-characterized tumor-associated antigens (TAAs), matching the peptide with a specific HLA allomorph, and also that the exogenous peptide-loaded HLA complex is only presented on the DC surface for a short period of time before arriving at a draining lymph node. ${ }^{70}$ This has led to the creation of several alternative antigen-loading strategies in the hope of filling all available HLA molecules with antigens.

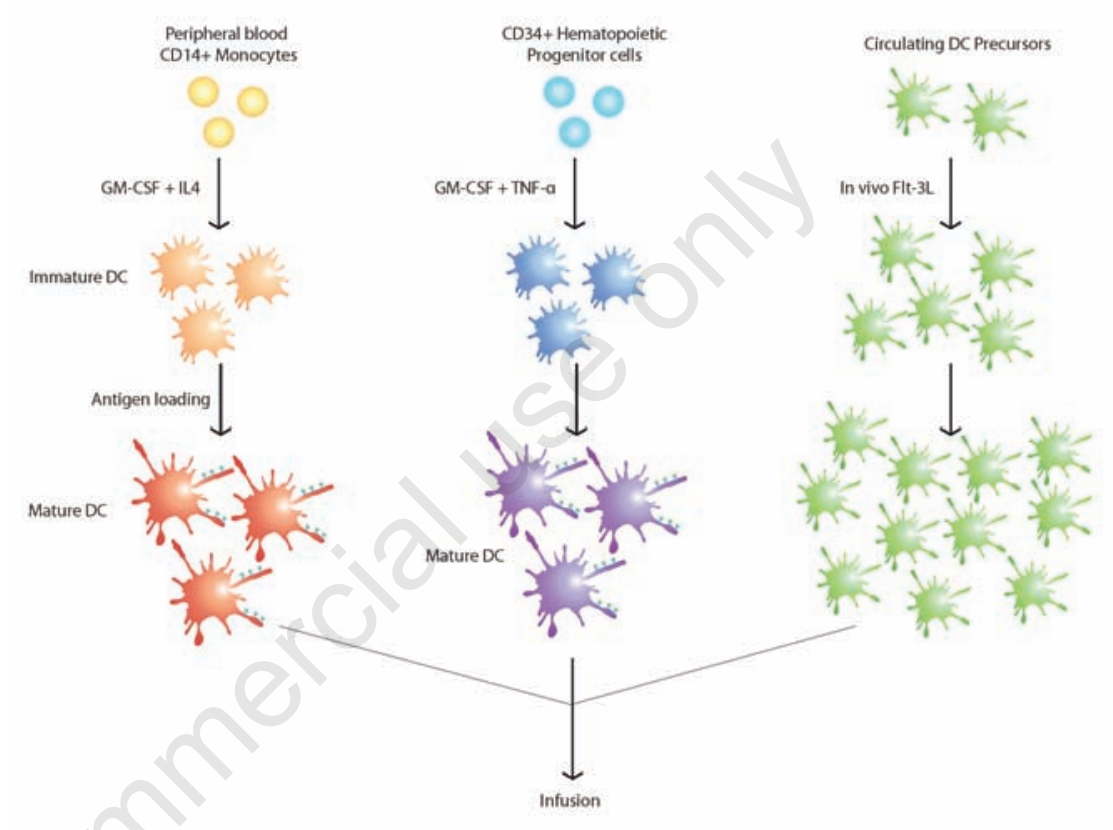

Figure 1. In vitro culture of peripheral blood $\mathrm{CD} 14^{+}$monocytes, $\mathrm{CD} 34^{+}$hematopoietic progenitor cells, and circulating dendritic cell (DC) precursors are the three main sources of mDCs used in clinical trials. Stimulation with key cytokines produce DCs ready for infusion.

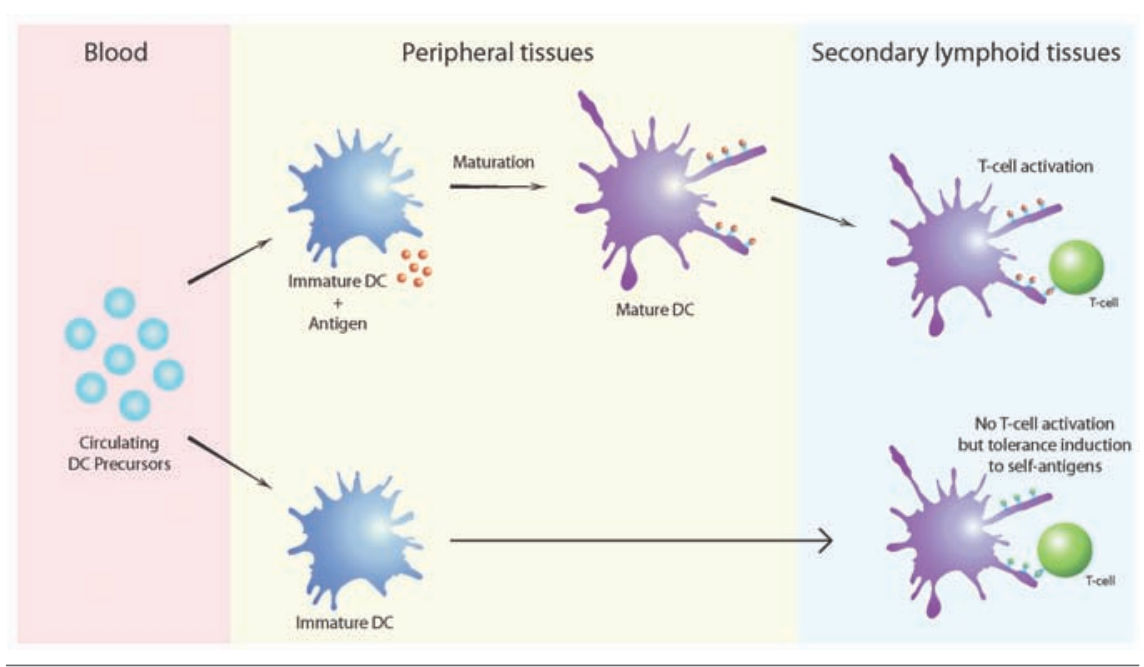

Figure 2. Antigen presentation by mature dendritic cells (DCs) activates naïve T lymphocytes while antigen presentation to naïv $T$ lymphocytes by immature DCs may induce tolerance rather than activation. 
One method aimed at improving the vaccines has been the use of proteins instead of peptides since these may result in multi-epitope presentation on the DC surface. Unfortunately this method has turned out not to be very immunogenic. Other techniques has also been tried, such as loading tumor RNA straight into the nucleus of DCs by a gene gun, or loading viral vectors with an antigen of choice, which is named gene transfer. ${ }^{71}$ Viral vectors such as adenoviruses or poxviruses have been used to treat colon carcinoma, and these can be loaded with tumor RNA by attaching the latter to the surface of the virosome and incorporating it inside the vesicles. ${ }^{72-73}$ When the viral vector is caught by the $\mathrm{DC}$, the surface antigen will be processed in endosomal compartments and presented by HLA class II molecules to $\mathrm{CD}^{+}{ }^{+} \mathrm{T}$ cells, whereas the RNA loaded inside the virosome infects the DC and is delivered into the nucleus. The RNA is then expressed and transported to the cytosol, where it is degraded into peptides by proteasomes. The peptides are further processed by the ER and Golgi apparatus and finally presented on the surface by HLA class I molecules to stimulate CD8+ T cells. By using this method, both HLA class I and HLA class II molecules are loaded and can present antigen at the cell surface, producing a more powerful and diverse immune response. In a set of clinical trials, recombinant viral vaccines have been shown to be more immunogenic than peptide and protein vaccines, and in addition the viral vectors can include genes that produce immunostimulatory cytokines such as IL-12 or co-stimulatory molecules such as B7.1. ${ }^{69,74-75}$

\section{Administration route}

One issue that still remains unclear is what administration route is the most favorable. In regard to CRC it is has been suggested that an intranodal injection of DCs could help bypass the negative effects of IL- 8 that may cause tumor retention of DCs. ${ }^{76}$ It has been proposed that injecting DCs produced ex vivo or even autologous DCs into a lymph node with the assistance of ultrasound could prevent this tumor retention of DCs. ${ }^{71}$

\section{Enhancing DCs in vivo}

Although DC vaccines produced in vitro can be administered through several routes, the DCs in these vaccines do not possess the same migratory efficiency as the DCs stimulated in vivo. For this reason, attempts have also been made to induce maturation of DCs in vivo, and one approach has been to incorporate antigens into monoclonal antibodies that target a specific DC surface molecule. ${ }^{72}$ Injecting this type of vaccine with an adjuvant may allow the DCs to be activated in a natural setting. By targeting the $\mathrm{CD} 8+\mathrm{cDCs}$, which possess the ability to present both types of HLA classes, the DCs may enhance both the cytotoxic T lymphocyte response and the antibody response.

\section{Discussion}

Almost 20 years ago Inaba et al. demonstrated that isolated DCs pulsed with antigen $e x$ vivo could be injected into mice and elicit an immune response against cells bearing the same antigen. ${ }^{73}$ The idea of ex vivo loading of DCs for vaccination purposes is indeed still highly attractive. Today the production of DC vaccines has shown that some of the immunosuppressive mechanisms induced by the tumor microenvironment - e.g., inhibition or polarization of DC generation, differentiation, and maturation - can be avoided by producing DCs ex vivo or enhancing the existing DCs in vivo. This may prevent DCs from inducing T cell tolerance or anergy. Breaking the suppressive microenvironment in the tumor has also been attempted by targeting the angiogenic VEGF, ID0, and other relevant cytokines such as MCSF, IL-6, and IL-10. These suppressive factors have mostly been targeted singly in studies, but instead a combination of several anti-monoclonal antibodies specifically targeting these substances could perhaps improve the outcome. Moreover, designing a vaccine that could inhibit as many of the immunosuppressive factors as possible would create a less hostile environment for the DCs in vivo, and this could therefore be an alternative to the timeconsuming ex vivo production of DCs. The questions still remain, however, of why many clinical trials have shown poor results and what role DC vaccines should be given in cancer therapy.

One vital factor that must be accounted for when discussing the lack of clinical effects is tumor burden. It is well known that in some cases activated effector cells are unable to infiltrate and destroy very large and solid tumors, and this may be the main reason why DC vaccines are not very eligible as primary antitumor therapy. Among 121 CRC patients subjected to DC therapy, none demonstrated clinical responses (partial or complete response), but 26 patients showed immune responses (detected by assays such as ELISPOT, tetramer staining, delayed-type hypersensitivity). ${ }^{77}$ The amount of tumor burden in each patient further complicates the process of comparing clinical results unless the patients are carefully grouped according to such factors as tumor grade, tumor size, and the presence of metastasis. In CRC the main tumor burden is conventionally removed surgically and the patient then undergoes chemotherapy. This may provide a better environment for DC vaccines even though cells of the immune system are also damaged by chemotherapy. In fact, one hypothesis is that DC vaccines injected after chemotherapy may even have the benefit of taking up greater amounts of available antigens from the debris produced by necrosis or apoptosis. ${ }^{78}$ Another advantage of chemotherapeutics such as cyclophosphamide might be the fact that they also inhibit T-regs, which could not only prevent ID0 expression but also suppress the known immunosuppressive secretion of cytokines IL-10 and TGF- $\beta$ by T-regs. Ultimately, therefore, chemotherapy might work synergistically with DC vaccines, and this could lead to a better activation of DCs and could prevent tolerance induced by T-regs.

A different kind of drug that might potentially produce synergistic effects with chemotherapy is the ID0 inhibitors. ID0 inhibitors of the 1-methyl-tryptophan (1MT) type consist of two different stereoisomers, the D- and the L-form. These two stereoisomers have been shown to have two different roles, since the 1MT L-form is a more potent inhibitor against IDO in general whereas the 1MT D-form is more effective at inhibiting IDO expression on tolerogenic DCs and is more effective when given in combination with chemotherapeutics. ${ }^{65}$ Hence, the DC dysfunction in CRC might benefit more from the D-form than the L-form of 1MT; but comparative studies are required to further explore this hypothesis.

MDSCs might also be eradicated by chemotherapy and the tolerance induced by TGF- $\beta$ and the inhibiting functions of reactive oxygen species (ROS), arginase, and NO could be reduced. ${ }^{79}$ However, other methods that target the immune-suppressing functions of MDSCs have been proposed, and results from on-going trials are awaited with great anticipation. One method is to deplete MDSCs from the organism by using monoclonal anti-Grl antibodies or a DNA vaccine targeting legumain, which is a protease overexpressed by MDSCs. Another proposed method is to pharmacologically inhibit the enzymes NOS and arginase by the PDE-5 inhibitor sildenafil or inhibit ROS by ROS inhibitors. ${ }^{14} \mathrm{An}$ attractive idea for inhibition of MDSCs is to use a combination of substances that could induce full maturation of the MDSCs into mature DCs, which would not only restrain the suppressing abilities of the MDSCs, but in fact provide the immune system with additional professional antigen-presenting cells to potentiate an even more powerful immune response. Therefore, creating a DC vaccine incorporating cytokines that would induce maturation of MDSCs to DCs would prevent the need for an anti-MDSC therapy and make MDSCs friends instead of foes.

There are many challenges regarding the design and production of the optimal DC vaccine. Which antigens should be used may depend on both the individual and the tumor type, but preferably the antigen should be 
expressed in a significant amount on the tumor, so that the immune response should target the tumor cells but not normal cells. Using self-proteins as a source of antigen makes it essential to evaluate the risk of autoimmune reactions. In CRC the carcinoembryonic antigen (CEA) is often chosen as a target since this antigen is heavily overexpressed in tumor tissue, although it is also expressed, but in much lower amounts, in normal tissue such as colonic crypts. ${ }^{75}$ Several clinical trials targeting this antigen in patients injected with DC vaccines did not show any serious autoimmune or toxic effects, which support the theory that DC-based therapy is a relatively safe method in cancer immunotherapy ${ }^{80}$ Another hope for the future to achieve successful treatment of CRC is to find more tumor-specific antigens. Four nuclear matrix proteins have already been found: colon cancer-specific antigens $2-5$, which, as the names imply, are specifically expressed in CRC. ${ }^{81}$ Besides the fact that these antigens could be potential tumor markers and help in the diagnosis of $\mathrm{CRC}$ at an early stage or at disease recurrence, they also present an opportunity of specifically targeting the malignant cells.

The frequent impairments, whether direct or indirect, of HLA class I expression in CRC are good examples of tumor evasion. ${ }^{14}$ The qualitatively and quantitatively altered peptide repertoire presented by these tumor cells may be significantly different from peptides presented by cells with intact APM and HLA class I expression, such as DCs. This strongly suggests that HLA class I expression should be considered when developing DC-based therapies. When it comes to adjuvants, the knowledge obtained from the tumor-evading techniques in CRC raises a very important question, which is whether we are still to regard IFN- $\gamma$ as a critical player in antitumor therapy or not. If IFN- $\gamma$ does prevent T cell proliferation and differentiation as a result of ID0 upregulation, and activates MDSCs, perhaps its beneficial effects such as activating macrophages and improving their HLA class I and II expression might not outweigh the negative effects. Further comparative results are needed to decide whether IFN- $\gamma$ should still be used as an adjuvant or not. Another drug considered as a potential adjuvant for the treatment of metastatic CRC in the future is bevacizumab. This drug consists of monoclonal antibodies that bind to the VEGF receptors and thereby inhibit tumor angiogenesis and recruitment of progenitor cells from the bone marrow. ${ }^{82}$ Bevacizumab is an FDA-approved drug and its effectiveness as a monotherapy has already been documented. ${ }^{82}$ However, one fundamental reason for the prospective use of bevacizumab as an adjuvant is because the effects that normalize tumor vasculature could possibly enhance the delivery of chemotherapeutics to tumors, which ultimately would improve the effects of the chemotherapy. However, the combination of bevacizumab with chemotherapy as a potential treatment in CRC still needs to be refined, because of several side effects and because the greatest effect of bevacizumab is seen above the maximum tolerable dose. ${ }^{83}$

\section{Conclusions}

Several studies using different models and species have shown how the tumor environment inflicts its inhibiting effects on the differentiation of progenitor cells to DCs as well as on DCs per se, and how this results in markedly reduced immune responses against malignant cells. The knowledge gained from the characterization and understanding of these complex immunosuppressive mechanisms enables us to circumvent some of these negative effects through vaccine design and administration. However, to date DC-based vaccines have shown limited clinical success, probably due to a combination of tumor burden and the fact that many things remain to be understood about the tumor environment and DC biology. Thus, identification of tumorderived substances and strategies to block these are important if the therapeutic qualities and outcomes of DC vaccines are to be enhanced. There is still a great deal of room for improvement of DC-based therapy, and at this point $\mathrm{DC}$ vaccines may as yet be inadequate to stand alone in cancer therapy; but they are a highly attractive therapy in combination with other therapeutic methods. For further improvements of DC-based therapies it is of the utmost importance that the methods used to define clinical effects follow strict guidelines so as to ensure objectivity and provide a basis for valid results that can be used in cancer research. Better efficacy of the DC vaccines, combined with their relatively safe and non-toxic characteristics, may therefore give the DCs an interesting role in combination therapy with other modalities such as chemotherapy, radiotherapy, or even other types of immunotherapy in the future.

\section{References}

1. Liu YJ. IPC: professional type 1 interferonproducing cells and plasmacytoid dendritic cell precursors. Annu Rev Immunol 2005;23:275-306.

2. Takahashi T, Tagami T, Yamazaki S et al. Immunologic self-tolerance maintained by CD25(+)CD4(+) regulatory T cells constitutively expressing cytotoxic T lympho- cyte-associated antigen 4. J Exp Med 2000;192:303-10.

3. Yuan J, Gnjatic S, Li H et al. CTLA-4 blockade enhances polyfunctional NY-ESO-1 specific $\mathrm{T}$ cell responses in metastatic melanoma patients with clinical benefit. Proc Natl Acad Sci USA 2008;105:20410-5.

4. Mayordomo JI, Zorina T, Storkus WJ et al. Bone marrow-derived dendritic cells serve as potent adjuvants for peptide-based antitumor vaccines. Stem Cells 1997;15:94103.

5. Ambe K, Mori M, Enjoji M. S-100 proteinpositive dendritic cells in colorectal adenocarcinomas. Distribution and relation to the clinical prognosis. Cancer 1989;63: 496-503.

6. Gabrilovich DI, Corak J, Ciernik IF et al. Decreased antigen presentation by dendritic cells in patients with breast cancer. Clin Cancer Res 1997;3:483-90.

7. Satthaporn S, Robins A, Vassanasiri W et al. Dendritic cells are dysfunctional in patients with operable breast cancer. Cancer Immunol Immunother 2004;53: 510-8.

8. Babatz J, Rollig C, Lobel B et al. Induction of cellular immune responses against carcinoembryonic antigen in patients with metastatic tumors after vaccination with altered peptide ligand-loaded dendritic cells. Cancer Immunol Immunother 2006;55:268-76.

9. Huerta S, Goulet EJ, Livingston EH. Colon cancer and apoptosis. Am J Surg 2006;191: 517-26.

10. Jemal A, Siegel R, Xu J, Ward E. Cancer statistics, 2010. CA Cancer J Clin 2010;60: 277-300.

11. Jemal A, Murray T, Ward E et al. Cancer statistics, 2005. CA Cancer J Clin 2005;55:10-30.

12. Blanes A, Diaz-Cano SJ. Complementary analysis of microsatellite tumor profile and mismatch repair defects in colorectal carcinomas. World J Gastroenterol 2006;12:5932-40.

13. Maleno I, Cabrera CM, Cabrera T et al. Distribution of HLA class I altered phenotypes in colorectal carcinomas: high frequency of HLA haplotype loss associated with loss of heterozygosity in chromosome region $6 \mathrm{p} 21$. Immunogenetics 2004;56: 244-53.

14. Cabrera T, Collado A, Fernandez MA et al. High frequency of altered HLA class I phenotypes in invasive colorectal carcinomas. Tissue antigens 1998;52:114-23.

15. Cabrera CM, Lopez-Nevot MA, Jimenez P, Garrido F. Involvement of the chaperone tapasin in HLA-B44 allelic losses in colorectal tumors. Int J Cancer 2005;113:6118.

16. Cabrera CM, Jimenez P, Cabrera T et al. 
Total loss of MHC class I in colorectal tumors can be explained by two molecular pathways: beta2-microglobulin inactivation in MSI-positive tumors and LMP7/TAP2 downregulation in MSI-negative tumors. Tissue antigens 2003;61:2119.

17. Cabrera T, Maleno I, Lopez-Nevot MA et al. High frequency of HLA-B44 allelic losses in human solid tumors. Hum immunol 2003;64:941-50.

18. Dierssen JW, de Miranda NF, Ferrone S et al. HNPCC versus sporadic microsatelliteunstable colon cancers follow different routes toward loss of HLA class I expression. BMC Cancer 2007;7:33.

19. Reis e Sousa C, Germain RN. Major histocompatibility complex class I presentation of peptides derived from soluble exogenous antigen by a subset of cells engaged in phagocytosis. J Exp Med 1995;182:84151.

20. Yanagihara S, Komura E, Nagafune J et al. EBI1/CCR7 is a new member of dendritic cell chemokine receptor that is up-regulated upon maturation. J Immunol 1998;161:3096-102.

21. Seth S, Oberdorfer L, Hyde R et al. CCR7 essentially contributes to the homing of plasmacytoid dendritic cells to lymph nodes under steady-state as well as nflammatory conditions. J Immunol 2011;186: 3364-72.

22. Gunn MD, Tangemann K, Tam C et al. A chemokine expressed in lymphoid high endothelial venules promotes the adhesion and chemotaxis of naive T lymphocytes. Proc Natl Acad Sci USA 1998;95:25863.

23. Gunn MD, Kyuwa S, Tam C et al. Mice lacking expression of secondary lymphoid organ chemokine have defects in lymphocyte homing and dendritic cell localization. J Exp Med 1999;189:451-60.

24. Luther SA, Tang HL, Hyman PL et al. Coexpression of the chemokines ELC and SLC by T zone stromal cells and deletion of the ELC gene in the plt/plt mouse. Proc Natl Acad Sci USA 2000;97:12694-9.

25. Shortman K, Liu YJ. Mouse and human dendritic cell subtypes. Nat Rev Immunol 2002;2:151-61.

26. Segura E, Villadangos JA. Antigen presentation by dendritic cells in vivo. Curr Opin Immunol 2009;21:105-10.

27. Wu L, D'Amico A, Hochrein $\mathrm{H}$ et al. Development of thymic and splenic dendritic cell populations from different hemopoietic precursors. Blood 2001;98: 3376-82.

28. Traver D, Akashi K, Manz M et al. Development of CD8alpha-positive dendritic cells from a common myeloid progenitor. Science 2000;290:2152-4.
29. McKenna K, Beignon AS, Bhardwaj N. Plasmacytoid dendritic cells: linking innate and adaptive immunity. J Virol 2005;79:17-27.

30. Vanbervliet B, Bendriss-Vermare $\mathrm{N}$, Massacrier $\mathrm{C}$ et al. The inducible CXCR3 ligands control plasmacytoid dendritic cell responsiveness to the constitutive chemokine stromal cell-derived factor 1 (SDF-1)/CXCL12. J Exp Med 2003;198:82330.

31. Inaba K, Schuler G, Witmer MD et al. Immunologic properties of purified epidermal Langerhans cells. Distinct requirements for stimulation of unprimed and sensitized T lymphocytes. J Exp Med 1986;164:605-13.

32. Grouard G, Durand I, Filgueira L et al. Dendritic cells capable of stimulating $\mathrm{T}$ cells in germinal centres. Nature 1996;384:364-7.

33. Hu M, Polyak K. Microenvironmental regulation of cancer development. Curr Opin Genet Dev 2008;18:27-34.

34. Matsushima K, Larsen CG, DuBois GC, Oppenheim JJ. Purification and characterization of a novel monocyte chemotactic and activating factor produced by a human myelomonocytic cell line. J Exp Med 1989;169:1485-90.

35. Coussens LM, Werb Z. Inflammation and cancer. Nature 2002;420:860-7.

36. Bunt SK, Yang L, Sinha P et al. Reduced inflammation in the tumor microenvironment delays the accumulation of myeloidderived suppressor cells and limits tumor progression. Cancer Res 2007;67:1001926.

37. Brigati C, Noonan DM, Albini A, Benelli R. Tumors and inflammatory infiltrates: friends or foes? Clin Exp Metastasis 2002;19:247-58.

38. Gabrilovich DI, Bronte V, Chen SH et al. The terminology issue for myeloid-derived suppressor cells. Cancer Res 2007;67:425.

39. Gabrilovich DI, Nagaraj S. Myeloid-derived suppressor cells as regulators of the immune system. Nat Rev Immunol 2009;9:162-74.

40. Iqbal S, Lenz HJ. Integration of novel agents in the treatment of colorectal cancer. Cancer chemother Pharmacol 2004;54Suppl1:S32-9.

41. Dikov MM, Oyama T, Cheng $P$ et al. Vascular endothelial growth factor effects on nuclear factor-kappaB activation in hematopoietic progenitor cells. Cancer Res 2001;61:2015-21.

42. Ohm JE, Carbone DP. VEGF as a mediator of tumor-associated immunodeficiency. Imm Res 2001;23:263-72.

43. Bennaceur K, Chapman JA, Touraine JL, Portoukalian J. Immunosuppressive networks in the tumour environment and their effect in dendritic cells. Biochim Biophys Acta 2009;1795:16-24.

44. Gabrilovich DI, Cheng P, Fan Y et al. H1(0) histone and differentiation of dendritic cells. A molecular target for tumor-derived factors. J Leukoc Biol 2002;72:285-96.

45. Sabat R, Asadullah K. Interleukin-10 in Cancer Immunity. In: Cancer Immune Therapie: Current and Future Strategies. Stuhler G, Walden P ed. Weinheim; 2002. p.155.

46. Buelens C, Verhasselt V, De Groote D et al. Interleukin-10 prevents the generation of dendritic cells from human peripheral blood mononuclear cells cultured with interleukin-4 and granulocytemacrophage-colony-stimulating factor. Eur J Immunol 1997;27:756-62.

47. Fricke I, Gabrilovich DI. Dendritic cells and tumor microenvironment: a dangerous liaison. Immunol Ivest 2006;35:459-83.

48. Shurin MR, Yurkovetsky ZR, Tourkova IL et al. Inhibition of CD40 expression and CD40-mediated dendritic cell function by tumor-derived IL-10. Int $\mathrm{J}$ Cancer 2002;101:61-8.

49. Mocellin S, Panelli MC, Wang E et al. The dual role of IL-10. Trends Immunol 2003;24:36-43.

50. Mocellin S, Marincola FM, Young HA. Interleukin-10 and the immune response against cancer: a counterpoint. J Leukoc Biol 2005;78:1043-51.

51. Brew R, Erikson JS, West DC et al. Interleukin- 8 as an autocrine growth factor for human colon carcinoma cells in vitro. Cytokine 2000;12:78-85.

52. Wilson AJ, Byron K, Gibson PR. Interleukin-8 stimulates the migration of human colonic epithelial cells in vitro. Clin Sci 1999;97:385-90.

53. Eckmann L, Jung HC, Schurer-Maly C et al. Differential cytokine expression by human intestinal epithelial cell lines: regulated expression of interleukin 8. Gastroenterol 1993;105:1689-97.

54. Li A, Varney ML, Singh RK. Expression of interleukin 8 and its receptors in human colon carcinoma cells with different metastatic potentials. Clin Cancer Res 2001;7:3298-304.

55. Rubie C, Frick VO, Pfeil Sr 0 et al. Correlation of IL-8 with induction, progression and metastatic potential of colorectal cancer. World J Gastroenterol 2007;13:4996-5002.

56. Feijoo E, Alfaro C, Mazzolini G et al. Dendritic cells delivered inside human carcinomas are sequestered by interleukin-8. Int J Cancer 2005;116:275-81.

57. Stanley ER, Heard PM. Factors regulating macrophage production and growth. Purification and some properties of the colony stimulating factor from medium 
conditioned by mouse L cells. J Biol Chem 1977;252:4305-12.

58. Zins K, Abraham D, Sioud M, Aharinejad S. Colon cancer cell-derived tumor necrosis factor-alpha mediates the tumor growthpromoting response in macrophages by up-regulating the colony-stimulating factor-1 pathway. Cancer Res 2007;67:103845.

59. Menetrier-Caux C, Montmain G, Dieu MC et al. Inhibition of the differentiation of dendritic cells from CD34(+) progenitors by tumor cells: role of interleukin- 6 and macrophage colony-stimulating factor. Blood 1998;92:4778-91.

60. Menetrier-Caux C, Thomachot MC, Alberti L, Montmain G, Blay JY. IL-4 prevents the blockade of dendritic cell differentiation induced by tumor cells. Cancer Res 2001;61:3096-104.

61. Park SJ, Nakagawa T, Kitamura H et al. IL6 regulates in vivo dendritic cell differentiation through STAT3 activation. J Immunol 2004;173:3844-54.

62. Mellor AL, Munn DH. ID0 expression by dendritic cells: tolerance and tryptophan catabolism. Nat Rev Immunol 2004;4:76274.

63. Daubener W, MacKenzie CR. IFN-gamma activated indoleamine 2,3-dioxygenase activity in human cells is an antiparasitic and an antibacterial effector mechanism. Adv Exp Med Biol 1999;467:517-24.

64. Brandacher G, Perathoner A, Ladurner R et al. Prognostic value of indoleamine 2,3dioxygenase expression in colorectal cancer: effect on tumor-infiltrating T cells. Clin Cancer Res 2006;12:1144-51.
65. Katz JB, Muller AJ, Prendergast GC. Indoleamine 2,3-dioxygenase in T-cell tolerance and tumoral immune escape. Immunol Rev 2008;222:206-21.

66. Palucka AK, Ueno H, Fay JW, Banchereau J. Taming cancer by inducing immunity via dendritic cells. Immunol Rev 2007;220:129-50.

67. Ballestrero A, Boy D, Moran E et al. Immunotherapy with dendritic cells for cancer. Adv Drug Deliv Rev 2008;60:17383.

68. Reis e Sousa C. Dendritic cells in a mature age. Nat Rev Immunol 2006;6:476-83.

69. Mazzolini G, Murillo 0, Atorrasagasti C et al. Immunotherapy and immunoescape in colorectal cancer. World J Gastroenterol 2007;13:5822-31.

70. Kikuchi T. Genetically modified dendritic cells for therapeutic immunity. Tohoku J Exp Med 2006;208:1-8.

71. Engell-Noerregaard L, Hansen TH, Andersen MH et al. Review of clinical studies on dendritic cell-based vaccination of patients with malignant melanoma: assessment of correlation between clinical response and vaccine parameters. Cancer Immunol Immunother 2009;58:1-14.

72. Shortman K, Lahoud MH, Caminschi I. Improving vaccines by targeting antigens to dendritic cells. Exp Mol Med 2009;41:616.

73. Inaba K, Metlay JP, Crowley MT, Steinman RM. Dendritic cells pulsed with protein antigens in vitro can prime antigen-specific, MHC-restricted T cells in situ. J Exp Med 1990;172:631-40.

74. Zeh HJ, Stavely-0'Carroll K, Choti MA.
Vaccines for colorectal cancer. Trends Mol Med 2001;7:307-13.

75. Smith CL, Dulphy N, Salio M, Cerundolo V. Immunotherapy of colorectal cancer. $\mathrm{Br}$ Med Bull 2002;64:181-200.

76. Cranmer LD, Trevor KT, Hersh EM. Clinical applications of dendritic cell vaccination in the treatment of cancer. Cancer Immunol Immunother 2004;53:275-306.

77. Burgdorf SK. Dendritic cell vaccination of patients with metas- tatic colorectal cancer. Dan Med Bull 2010;57:B4171.

78. Melief CJ. Cancer immunotherapy by dendritic cells. Immunity 2008;29:372-83.

79. Ko JS, Zea AH, Rini BI et al. Sunitinib mediates reversal of myeloid-derived suppressor cell accumulation in renal cell carcinoma patients. Clin Cancer Res 2009;15: 2148-57.

80. Reddy GK. The role of immunotherapy in the treatment of colorectal cancer. Clin Colorectal Cancer 2006;5:324-6.

81. Walgenbach-Brunagel G, Burger B, Leman ES et al. The use of a colon cancer associated nuclear antigen CCSA-2 for the blood based detection of colon cancer. J Cell Biochem 2008;104:286-94.

82. Mulder K, Scarfe A, Chua N, Spratlin J. The role of bevacizumab in colorectal cancer: understanding its benefits and limitations. Expert Opin Biol Ther 2011;11:40513.

83. Chase JL. Clinical use of anti-vascular endothelial growth factor monoclonal antibodies in metastatic colorectal cancer. Pharmacotherapy 2008;28:23S-30S. 